\title{
Insertion of Pause in Drawing from Babbling for Robot's Developmental Imitation Learning
}

\author{
Shun Nishide ${ }^{1}$, Keita Mochizuki ${ }^{2}$, Hiroshi G. Okuno ${ }^{2}$, and Tetsuya Ogata ${ }^{3}$
}

\begin{abstract}
In this paper, we present a method to improve a robot's imitation performance in a drawing scenario by inserting pauses in motion. Human's drawing skills are said to develop through five stages: 1) Scribbling, 2) Fortuitous Realism, 3) Failed Realism, 4) Intellectual Realism, and 5) Visual Realism. We focus on stages 1) and 3) for creating our system, each corresponding to body babbling and imitation learning, respectively. For stage 1), the robot randomly moves its arm to associate robot's arm dynamics with the drawing result. Presuming that the robot has no knowledge about its own dynamics, the robot learns its body dynamics in this stage. For stage 3), we consider a scenario where a robot would imitate a human's drawing motion. Upon creating the system, we focus on the motionese phenomenon, which is one of the key factors for discussing acquisition of a skill through a human parent-child interaction. In motionese, the parent would first show each action elaborately to the child, when teaching a skill. As the child starts to improve, the parent's actions would be simplified. Likewise in our scenario, the human would first insert pauses during the drawing motions where the direction of drawing changes (i.e. corners). As the robot's imitation learning of drawing converges, the human would change to drawing without pauses. The experimental results show that insertion of pause in drawing imitation scenarios greatly improves the robot's drawing performance.
\end{abstract}

\section{INTRODUCTION}

Imitation learning [1] in human-robot interaction is considered as one of the effective approaches for robot's skill acquisition in the cognitive developmental robotics field [2]. In human development, the function of imitation can also be observed in the early ages of infants [3]. Many work on imitation learning for robots have been conducted. Arie et al. focused on a robot model that imitates human motions through primitives of motion [4]. Demirls et al. created a robot imitation system from motor babbling using a local representation model [5]. Calinon et al. created a learning by imitation system, which extracts relevant features of a given task, to learn and generalize robot motions [6]. Yokoya et al. focused on imitation by prediction of other individuals through projection of the robot's own body dynamics model [7]. Most of these studies considered imitation strategy only from the robot's perspective, and do not discuss about the human's motions.

\footnotetext{
${ }^{1}$ Shun Nishide is with the Hakubi Center for Advanced Researches, Kyoto University, 606-8501 Kyoto, Japan nishideekuis.kyoto-u.ac.jp

${ }^{2}$ Keita Mochizuki and Hiroshi G. Okuno are with the Graduate School of Informatics, Kyoto University, 606-8501 Kyoto, Japan \{motizuki, okuno\} akuis. kyoto-u.ac.jp

${ }^{3}$ Tetsuya Ogata is with the Department of Intermedia Art and Science, Waseda University, 169-8555 Tokyo, Japan ogata@waseda.jp
}

In this paper, we introduce a mutual imitation strategy between a human and robot for improving the robot's imitation performance. In consideration of the approach, a phenomenon called motionese is a key factor seen in imitation learning between a parent and a child [10]. When a parent starts to teach the child a skill composed of a series of actions, the parent tends to show each action elaborately or emphatically. As the child starts to get used to the actions, the parent would simplify the teaching motion. This phenomenon, motionese, is said to facilitate the developmental process. Nagai et al. has also discussed about the effects of motionese to imitation learning between human and robot [11]. In spite of the attraction of the motionese phenomenon, few studies have actually adapted the approach to real robot platforms.

In our previous paper, we created a robot's imitation of human's shape drawing [9]. The drawing scenario is a good example that greatly involves physical embodiment while the result is easy to analyze. The model was based on stages 1) and 3) of the definition of human infant's development of drawing skills by Louquet [8].

1) Scribbling (1-3 yrs)

2) Fortuitous Realism (2-4 y rs)

3) Failed Realism (3-7 yrs)

4) Intellectual Realism (4-8 yrs)

5) Visual Realism $(8+y r s)$

In 1), an infant moves its arm randomly, drawing shapes of less significance. In this stage, the infant learns the relationship between its body dynamics and the shapes drawn. In 2), the infant exploits the similarity between the drawn shape and objects in the real world, raising the motivation towards imitation. In 3), the infant draws incomplete shapes by copying objects in view, due to lack of physical drawing abilities. In 4), the infant draws objects that come to mind. In 5), the infant completely copies objects in view.

In this paper, we improve the interaction model based on the motionese phenomenon by changing the human motion as the robot develops. In particular, first the human would pause his actions at the start of drawing and at corners of the shapes. The pause implies that the action (direction of drawing motion) would change. After the robot's imitation learning of drawing has converged, the human would draw the same shapes without pauses. In our experiment, we first show that inserting pauses improves the robot's drawing skills of basic shapes. We then show the effects of changing human's actions (from motion with pause to motion without pause) during the interaction to the robot's imitation perfor- 
mance.

Drawing robots based on engineering approaches have also been proposed. Kudoh et al. succeeded in drawing with a grasped pen in a robot arm by acquiring a threedimensional model of a target in real world using a stereo camera, extracting the features of the target, and drawing by calculating the inverse kinematics [12]. Kulvicius et al. focused on joining trajectories by modifying the dynamic movement primitive formulation, reproducing the target trajectory with high precision [13]. These work have created highly sophisticated systems with various potentials to applications. On the other hand, we focus on acquisition of drawing skills based on human development, constructing the system from scratch (babbling), whereas previous work on drawing imitation often assumed manual predesigning of the systems to some extent.

The rest of the paper is composed as follows. In Section II, we present the construction of the learning model. In Section III, we present the developmental human-robot imitation learning system. In Section IV, the setup of the experiment is presented. In Section $\mathrm{V}$, the results of the experiment are presented. In Section VI, discussions of the results are presented. Conclusions and future work are presented in Section VII.

\section{OVERVIEW OF LEARNING MODEL}

As the learning model of the system, we utilize Multiple Timescales Recurrent Neural Network (MTRNN) [14], which is a variant of the Jordan-type recurrent neural network. MTRNN is composed of two layers of neurons, one representing the state of the current step $t$ (input layer) and the other representing the state of the next step $t+1$ (output layer). The two layers have identical groups of neurons so as the results of the output layer could be reinputted into the neurons in the input layer to calculate the state of step $t+2$. Therefore, MTRNN functions as a predictor to learn multiple nonlinear sequential patterns.

Each layer of MTRNN is composed of three groups of neurons: State neurons, Fast Context neurons $C_{F}$, and Slow Context neurons $C_{S}$, as shown in Fig. 1 . In this paper, the State neurons are divided into neurons representing Joint Angles (robot state) $R$ and Pen Positions (drawing state) $P$. Neurons are fully connected except between the State neurons $(R, P)$ and Slow Context neurons $\left(C_{S}\right)$, and Joint Angle neurons $(R)$ and Pen Position neurons $(P)$. Each neuron group possesses time constant values which represent how frequently the values of the neurons change along the sequence. Neurons with small time constants change rapidly, while those with large time constants change gradually. The time constants of State neurons are the smallest, those of Slow Context neurons are the largest, and those of Fast Context neurons are set in between the two. This composition of neuron groups with different time constants provides MTRNN the capability to learn the sequences by structuring the dynamics information. Therefore, MTRNN is capable of learning complex sequences compared to conventional recurrent neural network models.

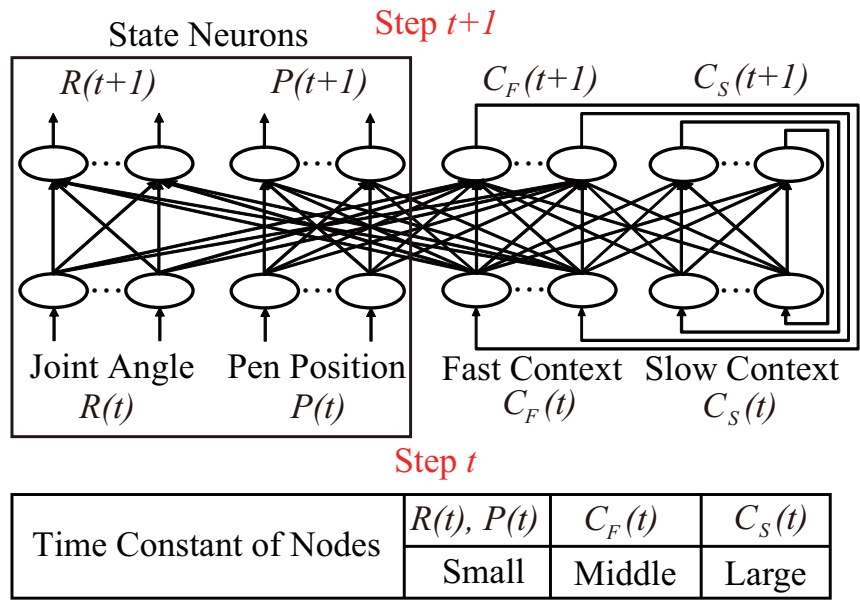

Fig. 1. Composition of MTRNN

MTRNN possesses three basic functions.

Training Input teaching sequences into the State neurons $(R, P)$ to update the weight values of the links between neurons and the initial values of $C_{F}$ and $C_{S}$ $\left(C_{F}(0)\right.$ and $\left.C_{S}(0)\right)$.

Generation Input $C_{F}(0)$ and $C_{S}(0)$ to calculate the sequence corresponding to the initial context values.

Recognition Input observed sequence into the State neurons $(R, P)$ to calculate $C_{F}(0)$ and $C_{S}(0)$ corresponding to the sequence.

\section{A. Training of MTRNN}

Training of MTRNN is done by computation of forward calculation and backward error propagation. Forward calculation inputs the sequence values of each step into MTRNN to calculate the outputs, which represent the predicted values of the next step. The errors of the final step are then propagated back to the initial step using Back Propagation Through Time (BPTT) algorithm [15] to update the weights of the network, $C_{F}(0)$ and $C_{S}(0)$. Forward calculation and backward error propagation are repetitively done through several thousands of calculation loops until training converges.

Forward calculation first calculates the internal values of the nodes and then calculates the output by applying the sigmoid function. The initial values of $C_{F}$ nodes and $C_{S}$ nodes $\left(C_{F}(0)\right.$ and $\left.C_{S}(0)\right)$ for the first calculation loop are randomly set. The internal value of the $i$ th neuron at step $t$ for the $n$th calculation loop, $u_{i, n}(t)$ is calculated by

$$
u_{i, n}(t)=\left(1-\frac{1}{\tau_{i}}\right) u_{i, n}(t-1)+\frac{1}{\tau_{i}}\left[\sum_{j \in N} w_{i j, n} x_{j, n}(t)\right]_{(1)}
$$

where $\tau_{i}$ is the time constant of the $i$ th neuron, $w_{i j, n}$ is the weight value from the $j$ th input neuron to the $i$ th output neuron for the $n$th calculation loop, and $x_{j, n}(t)$ is the input value. The output of the $i$ th neuron $y_{i, n}(t)$ is then calculated 
by applying the sigmoid function,

$$
y_{i, n}(t)=\operatorname{sigmoid}\left(-u_{i, n}(t)\right) .
$$

The teacher signals $T_{i}(t)$ are used for the input value $x_{i, n}(t)$ for the State neurons, while the output of the previous step $y_{i, n}(t-1)$ is used as the input value $x_{i, n}(t)$ for the Fast and Slow Context nodes.

After forward calculation, the BPT'T algorithm is used to update the weights of the network using the training error $E_{n}$. Training error $E_{n}$ is defined as the sum of squared output errors along the sequence, calculated as

$$
E_{n}=\sum_{t} \sum_{i}\left(y_{i, n}(t-1)-T_{i}(t)\right)^{2} .
$$

The weight from the $j$ th input to the $i$ th output is updated using the derivative of the training error $\partial E / \partial w_{i j}$ as

$$
w_{i j, n+1}=w_{i j, n}-\alpha \frac{\partial E_{n}}{\partial w_{i j, n}},
$$

where $\alpha$ is the training coefficient. $C_{F}(0)$ and $C_{S}(0)$ values are also updated in a similar manner as (4).

Forward calculation and backward error propagation are done repetitively by incrementing calculation loop $n$ until the training error converges. After training of MTRNN, a unique $C_{F}(0)$ and $C_{S}(0)$ value are acquired for each sequence. The $C_{F}(0)$ and $C_{S}(0)$ values correspond to a unique sequence and can be mutually calculated by recognition and generation functions presented in the next subsections.

\section{B. Generation with MTRNN}

Generation of sequences using MTRNN is a process to calculate a sequence from given $C_{F}(0)$ and $C_{S}(0)$ values. The generation process is conducted in a similar manner as the forward calculation of the training process. First, the initial State neuron value $(R(0), P(0))$ is input to calculate the next State neuron value $(R(1), P(1))$. These values $(R(1), P(1))$ are input into MTRNN to calculate $(R(2)$, $P(2))$. The calculation is done repetitively to calculate the whole sequence.

\section{Recognition with MTRNN}

Recognition of sequences using MTRNN is a process to calculate $C_{F}(0)$ and $C_{S}(0)$ values from a given sequence. The recognition process is conducted in a similar manner as the training process. Using the weight values of the trained MTRNN, a random $C_{F}(0)$ and $C_{S}(0)$ value is set as an initial value. MTRNN conducts forward calculation to calculate the output values by inputting the sequence, and propagates the errors back using the BPTT algorithm. Unlike the training process, recognition only updates the $C_{F}(0)$ and $C_{S}(0)$ values (the weights are fixed) during the calculation loop. Convergence of the calculation derives a unique $C_{F}(0)$ and $C_{S}(0)$ value which represents the sequence.

A characteristic of MTRNN is that a sequence comprising only the joint angle sequences (or only the pen position sequences) can be used for recognition. In this case, 0 is used as the output error for the missing sequence when back propagating errors. The missing sequence can also be recovered by applying the generation function after recognition.

\section{Developmental Human-Robot Imitation}

In this section, we present the developmental human-robot imitation system. The system comprises mainly from two phases.

Phase 1 Body Babbling

Phase 2 Incremental Imitation Learning

Phase 1 corresponds to 1) Scribbling presented in Section I. In this phase the robot obtains the relationship between the robot's joint angle dynamics and pen position dynamics. The robot randomly moves its arm acquiring the joint angle sequence and pen position sequence. The sequences are input into MTRNN for training. Through this approach, the model does not require manual predesigning of the system.

Phase 2 corresponds to 3) Failed Realism presented in Section II. In this phase, the human and robot take turns drawing and imitating based on the following algorithm.

Step 1 Human draws several shapes, showing it to the robot.

Step 2 Robot recognizes pen position sequences obtained in Step 1 and generates the joint angle sequence.

Step 3 Draw a shape by moving the robot's arm based on the joint angle sequence calculated by Step 2 .

Step 4 Calculate the squared error between the robot's drawing (pen position sequence) and human's drawing (pen position sequence).

Step 5 Select shapes with medial errors to retrain MTRNN. Step 6 Return to Step 2.

In the algorithm (Step 4 and 5), we select shapes with medial errors for incremental training of MTRNN. The aim of this is to reduce the effect of overfitting from shapes with small errors, and to accelerate the training process by neglecting those with large errors. As a result, the robot's drawing abilities of selected shapes impove, while those for other shapes decrease. The idea is based on "artificial curiosity" [16], where the robot's interest is focused not on completely predictable targets nor on inherently unpredictable targets, but on targets with learnable but yet unknown regularities. A diagram of the algorithm is shown in Fig. 2.

During the whole training process, the human changes the drawing motion based on the robot's imitation result. First, the human pauses his motion at the start of drawing and at the corners of shapes. When the human confirms that the robot improved in drawing corners of the shapes after several drawing imitations and retraining by the robot, the human draws the same shapes without pause at the start and at corners of the shapes. The robot continues to perform imitation drawing and retraining using the newly presented shapes.

\section{EXPERIMENTAL SETUP}

We conducted an experiment using the humanoid robot, NAO. During the experiment, NAO moved its arm using two DOFs (Shoulder:Roll and Elbow:Roll) grasping a digital pen. 


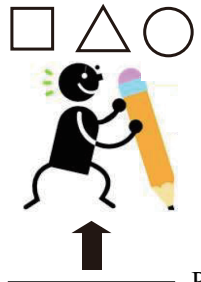

\section{MTRNN \\ (training)}
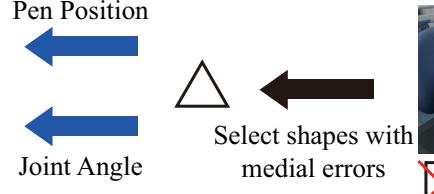

too hard.

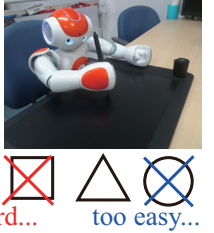

Fig. 2. Incremental Imitation Learning

The pen position was obtained using a pen tablet as a canvas when the pen was close to the tablet. The scene of NAO drawing on the pen tablet is shown in Fig. 3. The experiment is assumed that the robot and human are in the same position, not facing each other. Therefore, in this paper, we neglect the problem of projection of self, which is required when the robot should stand on the human's perspective.

The size of MTRNN is four State neurons (two for Joint Angle neurons and two for Pen Position neurons ( $\mathrm{x}$ and $\mathrm{y})$ ), $20 C_{F}$ neurons, and $8 C_{S}$ neurons. The time constants of State neurons, $C_{F}$ neurons, and $C_{S}$ neurons were set as 2, 5, 70, respectively. This composition of MTRNN was selected empirically based on several training results after body babbling training.

\section{A. Training Data Without Pause (Conventional Method)}

This subsection presents the detail of training data without insertion of pause in motion. The data is used for comparison of the proposed method.

\section{Phase 1: Body Babbling}

First, a random target joint angle was sent to NAO to move its arm in a constant velocity. After reaching the target joint angle, a new random target joint angle is sent. The procedure is conducted continuously. The drawing result during the motion is shown in Fig. 4. Note that the drawing is composed

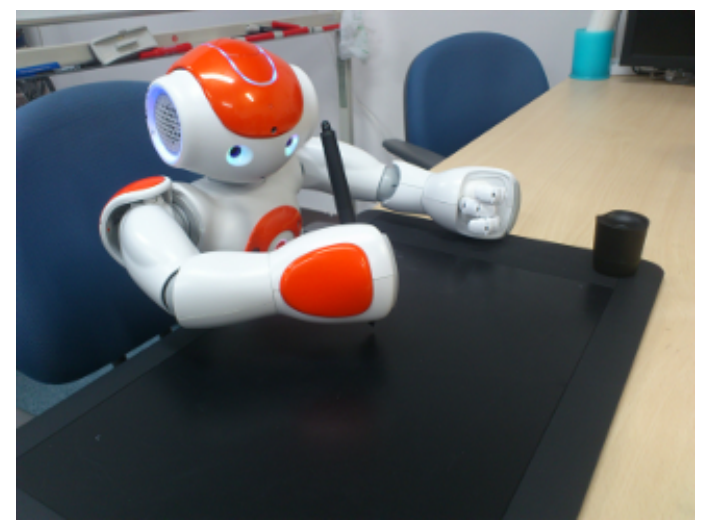

Fig. 3. Experiment Scene (Nao and Pen Tablet)

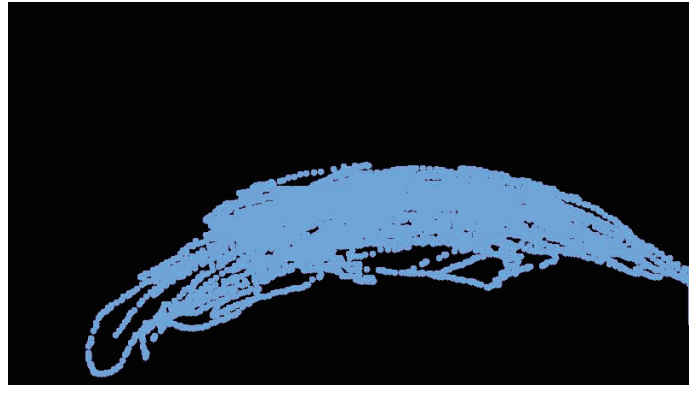

Fig. 4. Drawing Result on Pen Tablet by Robot's Body Babbling

mainly of arcs as NAO's arm was controlled by joint angles, not position. During the motion, the joint angle of NAO and the pen position is acquired at $30 \mathrm{fps}$. The whole sequence is divided into 80 sequences each comprising 100 steps.

Using an Intel(R) Core(TM) i7 processor (2.80 Ghz) with 4 cores, 4GB of memory, it took approximately 10 minutes to gather babbling data and 30 minutes to train MTRNN.

\section{Phase 2: Incremental Imitation Learning}

In this paper, we selected circle, triangle, and square for target shapes to draw. The shapes were drawn from four starting points for the circle and square, three for triangle, both in clockwise and anti-clockwise directions. A total of 22 motion sequences were acquired (left column of Fig. 5). In Step 5 of the imitation learning algorithm, seven data with medial errors were selected for retraining the model.

Using an Intel(R) Core(TM) i7 processor (1.90 Ghz) with 2 cores, 4GB of memory, it took approximately 15 minutes for training one cycle of the algorithm.

\section{B. Training Data with Pause (Proposed Method)}

For the proposed method, we modify the phases as follows.

\section{Phase 1: Body Babbling}

When sending a new target joint angle (when the robot's motion direction changes), a pause motion is inserted for 10 steps. The same drawing result is obtained as Fig. 4.

\section{Phase 2: Incremental Imitation Learning}

First, the human inserts a pause in the starting point and corners of squares and triangles for 10 steps when drawing the shapes (Step 1 of the imitation learning algorithm). After the robot's drawing performance converges, the same motions presented in the previous subsection without pause are shown for imitation.

\section{EXPERIMENTAL RESUlT}

The results of the drawing imitation experiment are shown in Fig. 5. Note that the drawing results are based on babbling experience and the robot does not have any knowledge about its body dynamics beforehand. The shapes on the left column represent the drawings that a human has shown. The numbers on the upper left corner of the shapes is the prefix number of the shape. The second column shows drawing results of the robot when the human has drawn the shapes with pause (proposed method). The third column shows drawing 


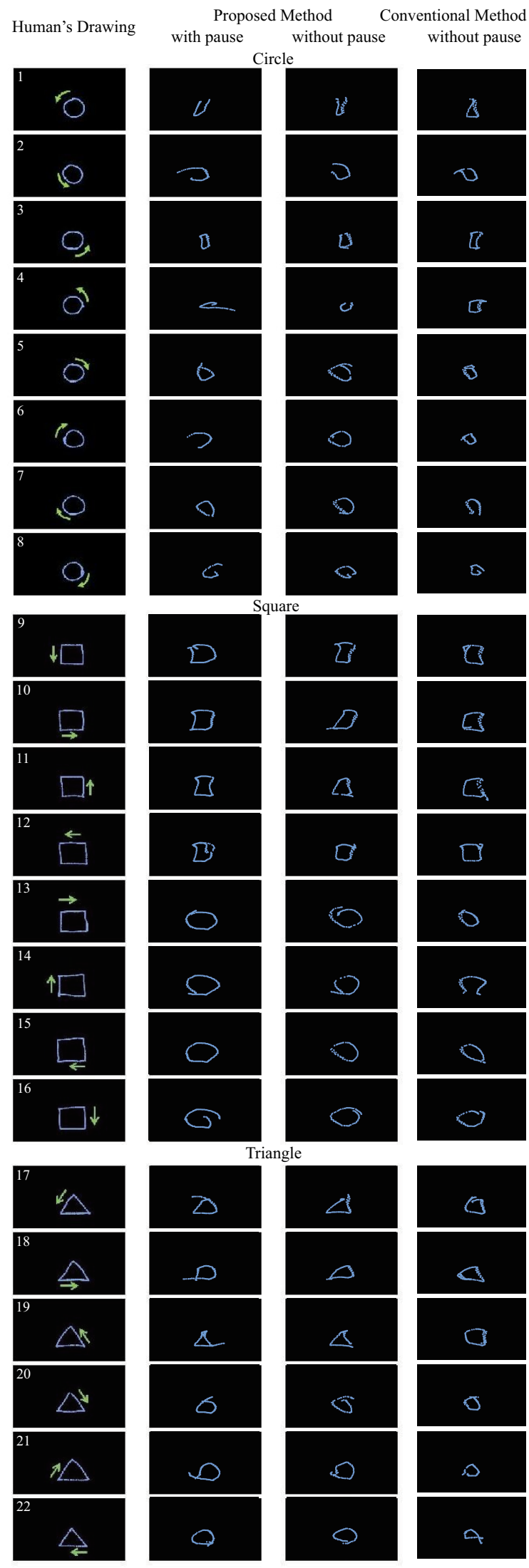

Fig. 5. Imitation Drawing Experiment Result results of the robot imitating human's motion without pause, after the robot has done imitation of human's motion with pause (proposed method). The fourth column shows drawing results of the robot imitating human's motion without pause (conventional method). Retraining was conducted six times for the proposed method and five times for the conventional method (training converged after that). The frequency each shape has been selected for retraining in the imitation algorithm are shown in Table I. From Table I and Fig. 5, it is notable that each shape was selected almost equally in the imitation algorithm.

Comparing the drawing performance imitation with and without pause in Fig. 5, it is notable that insertion of pause in motion greatly improves the imitation result. We evaluate the results quantitatively by two methods. First, we quantitatively evaluate the results of circles, by introducing a measure of Roundness [17] defined as,

$$
\begin{gathered}
\text { Roundness }=4 \pi S / L^{2} . \\
S: \text { Area of Shape } \\
L: \text { Perimeter of Shape }
\end{gathered}
$$

A value closer to 1.0 represents that the shape is closer to circle. The average Roundness values for the drawn shapes by the robot in Fig. 5 are shown in Table II. Table II shows that the proposed method with pause has better performance than conventional method for drawing circles. To evaluate the drawing performance of each shape, we calculate the error between the trajectory of the robot's drawing with human's drawing. The difference between the pen position of the robot and human are calculated and accumulated for every step. Table III shows the average error for each shape in each direction. In Table III, the letter in parenthesis after the shape represents the direction the shape has been drawn: (l) being anticlockwise and (r) being clockwise. From Table III, it is notable that insertion of pause greatly improves the drawing imitation performance of the robot.

Qualitatively evaluating the squares and triangles, it is notable that there is not much difference between the squares between the proposed method and conventional methods, but the performance of anti-clockwise drawing of triangles is better for the proposed method. In particular, IDs \#17 and \#19 for triangles were nicely drawn, even though they have not been retrained without pause in the proposed method. As the drawing of the two shapes are similar between the proposed method with and without pause, the results imply that the drawing performance of the proposed method without pause is also affected by previous training with pause. On the other hand, the performances of IDs \#9, \#10, and \#1 1 for squares seem to be slightly degraded for the proposed method without pause, though the features of squares are retained. The results of the experiment imply that the proposed method retains the previously trained experiences to some extent.

\section{DISCUSSION}

In our previous paper, we discussed the drawing performance comparison of clockwise and anti-clockwise shapes 
TABLE I

FREQUENCY OF S ELECTION FOR EACH S HA PE FOR RETRAINING IN Imitation Algorithm (Proposed, Conventional)

\begin{tabular}{|c|c||c|c||c|c|}
\hline \multicolumn{2}{|l|}{ Circle } & \multicolumn{2}{l|}{ Square } & \multicolumn{2}{l|}{ Triangle } \\
\hline Shape ID & \# times & Shape ID & \# times & Shape ID & \# times \\
\hline 1 & 1,2 & 9 & 1,1 & 17 & 0,1 \\
\hline 2 & 0,4 & 10 & 0,0 & 18 & 1,3 \\
\hline 3 & 2,2 & 11 & 0,2 & 19 & 0,1 \\
\hline 4 & 0,1 & 12 & 1,0 & 20 & 2,0 \\
\hline 5 & 3,2 & 13 & 5,4 & 21 & 2,1 \\
\hline 6 & 2,1 & 14 & 2,2 & 22 & 1,0 \\
\hline 7 & 3,1 & 15 & 5,1 & & \\
\hline 8 & 1,0 & 16 & 4,1 & & \\
\hline
\end{tabular}

TABLE II

Average Roundness Values

\begin{tabular}{|c||c|c|}
\hline & Anti-Clockwise & Clockwise \\
\hline Prop. (with pause) & 0.54 & 0.64 \\
\hline Prop. (first with, then w/o pause) & 0.62 & 0.60 \\
\hline Conv. (w/o pause) & 0.42 & 0.53 \\
\hline
\end{tabular}

(circles are better drawn clockwise while squares and triangles are better drawn anti-clockwise) [9]. In [9], better drawing of squares and triangles were related to robot's body babbling experience. The robot tended to draw squares and triangles in anti-clockwise direction using the motions generated during body babbling, while clockwise drawing of squares and triangles were motions not generated in body babbling. Better drawing of circles in clockwise direction was due to body structure, where human infants are also said to draw clockwise circles in the early stages of drawing development. Please refer to [9] for more detailed discussion about performance from the drawing direction aspect. In this paper, we focus on discussions about insertion of pause and motionese phenomenon.

\section{A. Insertion of Pause Motion for Imitation}

The results of the experiment showed great improvement of drawing imitation by inserting pauses in motion, specifically for circles and triangles. Pauses are often considered as moments for changing in motion primitives. Nagai et al. has shown that pauses were used to decompose long round sequences into several linear movements in teaching infants some action [18]. Analysis of Japanese traditional dance for robot imitation has shown that stopping postures exist between changes in motion [19]. We also assume that inserting pauses in motions assist MTRNN in recognizing a sequence in primitives.

In the experiment, we added pauses in the babbling motion, when a new joint angle command has been sent. We believe that this is a practical assumption as motion would usually stop when the direction of motion changes, not only for human infants but also for human adults. By training MTRNN with motion sequences with pauses, the robot would implicitly learn that a pause would tend to be a signal for change in motion direction. Therefore, imitation of human motions with pauses at corners were better performed as the robot could predict when to change its motions.
TABLE III

AVERAge TrajeCtOry ERROR FOR EACH SHAPE (IN CCENTIMETERS)

\begin{tabular}{|l|c|c|c|}
\hline & $\begin{array}{c}\text { (Proposed) } \\
\text { with pause }\end{array}$ & $\begin{array}{c}\text { (Proposed) } \\
\text { first with then w/o pause }\end{array}$ & $\begin{array}{c}\text { (Conventional) } \\
\text { without pause }\end{array}$ \\
\hline Circle(l) & 1.74 & 1.87 & 1.79 \\
\hline Circle(r) & 1.66 & 1.26 & 1.60 \\
\hline Square(l) & 1.28 & 2.18 & 1.57 \\
\hline Square(r) & 1.35 & 1.78 & 2.01 \\
\hline Triangle(l) & 1.20 & 1.03 & 1.55 \\
\hline Triangle(r) & 1.31 & 1.71 & 1.59 \\
\hline All & 1.42 & 1.64 & 1.68 \\
\hline
\end{tabular}

\section{B. Motionese Phenomenon for Imitation Learning}

In the experiment, qualitative and quantitative analysis of the result has shown that the proposed imitation learning system based on motionese phenomenon improves the performance of drawing. The proposed method first trains the imitation model using motions with pause. After switching to showing human motions without pause, the system would retrain MTRNN using the previously trained model. Therefore, the training would preserve the previous model while trying to adapt to the new data. Compared to training from scratch, the proposed method would achieve a better model though the training cost would be larger than conventional methods.

Other expressions of motionese phenomenon than pause (such as exaggerations) are also observed in interaction between a human adult and child. As we confirmed that pause would improve the imitation performance in humanrobot interaction, other expressions may also be effective in learning motion through imitation. Future work includes integration of our current system with other expressions of motionese phenomenon for creating a more sophisticated system.

\section{Remaining Issues Toward Online Interaction}

In this paper, we conducted experiments where the human and robot's drawing phases were completely separated as follows.

1) Robot babbling (robot's action)

2) Human drawing with pause (human's action)

3) Robot imitation of drawing for several trials (robot's action)

4) Human drawing without pause (human's action)

5) Robot imitation of drawing for several trials (robot's action)

As there are no intervenes when the other is acting, the current system requires improvement to a more seamless model. When concerning mutual interaction between humans, there are several interesting factors that should be considered. Perception ambiguity (difficulty to distinguish objects) is said to be one of the keys to trigger imitation of others [20]. During interaction, rhythm and time breaks play an important role to determine the timing of actions [21]. In addition to other phenomena in motionese described above, these systems should also be implemented to create 
a developmental human robot interaction system based on human development.

\section{CONCLUSION}

In this paper, a human-robot imitation learning system in a drawing scenario was presented. The main focus of the paper was insertion of pause in human's motion for robot's imitation performance improvement, and construction of the system based on the motionese phenomenon. We utilized MTRNN for creating the system and experimented it in a drawing imitation scenario where the robot starts from babbling with no knowledge about its body dynamics. The robot first draws by moving its arm randomly (babbling) to associate its arm joint angle dynamics with pen position dynamics. After babbling, a human presents several shapes by drawing it with a pause at corners. The robot calculates the arm joint motion from the human's pen position motion for imitating the drawing. Choosing the drawn shapes by the robot with medial errors from the human's for retraining data, the robot retrains MTRNN. After several loops of imitation, the robot's imitation converges. The human then presents the same shapes without pauses for the robot to imitate and retrain MTRNN in the same process. The experiment showed effectiveness of inserting pause in motions to improve the imitation performance. The performance of imitating motions without pause was also improved by the robot experiencing imitation of motions with pause beforehand.

Future work includes refining of the imitation algorithm for better human-robot imitation systems, and improving the system for practical applications. Further on, we plan to develop the imitation system to interaction systems, where the robot and human are required to act based on prediction of others. Projection of the self model, work such as [7], would also be required for the robot to stand on the human's perspective. We believe that our system would contribute to creating a smooth human-robot interaction system.

\section{ACKNOWLEDGMENT}

The work has been supported by JST PRESTO "Information Environment and Humans", MEXT Grant-in-Aid for Scientific Research on Innovative Areas "Constructive Developmental Science" (24119003), Grant-in-Aid for Young Scientists (B) (25730159), Kayamori Foundation of Informational Science Advancement, and Tateishi Science and Technology Foundation.

\section{REFERENCES}

[1] S. Schaal, "Is Imitation Learning the Route to Humanoid Robots?," Trends in Cognitive Sciences, Vol. 3, No. 6, pp. 233-242, 1999.
[2] M. Asada, K. MacDorman, H. Ishiguro, and Y. Kuniyoshi, "Cognitive developmental robotics as a new paradigm for the design of humanoid robots," Robotics and Autonomous Systems, vol. 37, pp. 185-193, 2001.

[3] A. N. Meltzoff and M. K. Moore, "Imitation of facial and manual gestures by human neonates," Science, Vol. 198, pp. 75-78, 1977.

[4] H. Arie, T. Arakaki, S. Sugano, and J. Tani, "Imitating others by composition of primitive actions: A neuro-dynamic model," Robotics and Autonomous Systems, Vol. 60, Issue 5, pp. 729-741, 2012.

[5] Y. Demirls and A. Dearden, "From motor babbling to hierarchical learning by imitation: a robot developmental pathway," Proc. of Int. Workshop on Epigenetic Robotics, pp. 31-37, 2005.

[6] S. Calinon, F. Guenter, and A. Billard, "On Learning, Representing and Generalizing a Task in a Humanoid Robot," IEEE Trans. on Systems, Man and Cybernetics, Vol. 37, Issue 2, pp. 286-298, 2007.

[7] R. Yokoya, T. Ogata, J. Tani, K. Komatani, and H. G. Okuno, "Discovery of Other Individuals by Projecting a Self-Model Through Imitation," Proc. of IEEE/RSJ Int. Conf. on Intelligent Robots and Systems, pp. 1009-1014, 2007.

[8] G. H. Louquet, "Le Dessin Enfantin," 1927.

[9] K. Mochizuki, S. Nishide, H. G. Okuno, and T. Ogata, "Developmental Human-Robot Imitation Learning of Drawing with a Neuro Dynamical System," Proc. of IEEE Int. Conf. on Systems, Man, and Cybernetics, 2013. (to appear)

[10] R. J. Brand, D. A. Baldwin, and L. A. Ashburn, "Evidence for 'motionese': modifications in mothers' infant-directed action," Developmental Science, Vol. 5, pp. 72-83, 2002.

[11] Y. Nagai and K. J. Rohlfing, "Computational Analysis of Motionese Toward Scaffolding Robot Action Learning," IEEE Trans. on Autonomous Mental Development, Vol. 1, No. 1, pp. 44-54, 2009.

[12] S. Kudoh, K. Ogawara, M. Ruchanurucks, and K. Ikeuchi, "Painting robot with multi-fingered hands and stereo vision," Robotics and Autonomous Systems, Vol. 57, No. 3, pp. 279-288, 2009.

[13] T. Kulvicius, K. Ning, M. Tamosiunaite, and F. Wörgötter, "Joining movement sequences: Modified dynamic movement primitives for robotics applications exemplified on handwriting," IEEE Trans. on Robotics, Vol. 28, Issue 1, pp. 145-157, 2012.

[14] Y. Yamashita and J. Tani, "Emergence of Functional Hierarchy in a Multiple Timescale Neural Network Model: a Humanoid Robot Experiment," PLoS Computational Biology, Vol. 4, No. 11, e1000220, 2008.

[15] P. Werbos, "Backpropagation through time: What it does and how to do it," Proc. of the IEEE, Vol. 78, No. 10, pp. 1550-1560, 2002.

[16] J. Schmidhuber, "A Possibility for Implementing Curiosity and Boredom in Model-Building Neural Controllers," in J. A. Meyer and S. W. Wilsonm editors, Proc. of the Int. Conf. on Simulation of Adaptive Behavior: From Animals to Animats, MIT Press/Bradford Books, pp. 222-227, 1991.

[17] “Sphericity," Wikipedia, http://en.wikipedia.org/wiki/Sphericity

[18] Y. Nagai, M. Asada, and K. Hosoda, "Learning for joint attention helped by functional development," Advanced Robotics, Vol. 20, Issue 10, pp. 1165-1181, 2006.

[19] S. Nakaoka, A. Nakazawa, K. Yokoi, H. Hirukawa, and K. Ikeuchi, "Generating Whole Body Motions for a Biped Humanoid Robot from Captured Human Dances," Proc. of IEEE Int. Conf. on Robotics and Automation, pp. 3905-3910, 2003.

[20] P. Andry, P. Gaussier, S. Moga, J. P. Banquet, J. Nadel, "Learning and communication via imitation: an autonomous robot perspective," IEEE Trans. on Systems, Man and Cybernetics, Vol. 31, Issue 5, pp. 431-442, 2001.

[21] P. Andry, A. Blanchard, and P. Gaussier, "Using the Rhythm of Nonverbal Human-Robot Interaction as a Signal for Learning," IEEE Trans. on Autonomous Mental Development, Vol. 3, No. 1, pp. 30-42, 2011. 